\section{AE E T}

ASOCIACIÓN ESPAÑOLA DE ECOLOGÍA TERRESTRE
Ecosistemas 21(3):97-99 [Septiembre-Diciembre 2012] Doi.: 10.7818/ECOS.2012.21-3.13

Artículo publicado en Open Access bajo los términos de Creative Commons attribution Non Comercial License.

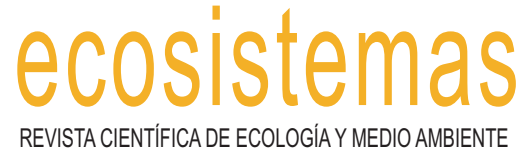

ISSN 1697-2473 / Open access

disponible en www.revistaecosistemas.net

\title{
Novedades en Ecosistemas
}

\author{
L. Cayuela ${ }^{1, *}$, Á. Alonso ${ }^{3}$, J.A. Blanco ${ }^{4}$, C. Puerta-Piñero ${ }^{5}$, S. Rodríguez-Echeverría ${ }^{6}, N^{\prime}$. Martín $^{1}$, L. Jiménez-Eguizabal ${ }^{2}$
}

(1) Departamento de Biología y Geología, ESCET, Universidad Rey Juan Carlos, c/ Tulipán s/n, 28933 Móstoles, Madrid, España

(2) Revista Ecosistemas. Asociación Española de Ecología Terrestre (AEET), Departamento de Biología y Geología, Universidad Rey Juan Carlos, c/ Tulipán s/n, 28933 Móstoles, Madrid, España.

(3) Departamento de Ciencias de la Vida, Unidad Docente de Ecología, Universidad de Alcalá, Crta. N-II Km 33,6 Campus Universitario, Edificio Biología, 28871 Alcalá de Henares, Madrid, España.

(4) Departamento de Ciencias del Medio Natural, ETSIA, Universidad Pública de Navarra, Campus de Arrosadía s/n, 31006, Pamplona, Navarra, España.

(5) CREAF, Centre de Recerca Ecològica I Aplicacions Forestals, Universidad Autónoma de Barcelona, Bellaterra, E-08193, España.

(6) Centro de Ecología Funcional. Departamento de Ciências da Vida. Universidad de Coimbra. 3000-455. Coimbra. Portugal.

*Autor de correpondencia: L. Cayuela [luis.cayuela@urjc.es]

Cayuela, L., Alonso, Á., Blanco, J.A., Puerta-Piñero, C., Rodríguez-Echeverría., Martín, N., Jiménez-Eguizábal, L. (2012). Novedades en Ecosistemas. Ecosistemas 21(3):97-99. Doi.: 10.7818/ECOS.2012.21-3.13

Comienza una nueva etapa de la revista Ecosistemas. Desde sus comienzos en formato electrónico, la revista ha contado con tres comités editoriales, al frente de los cuales han estado investigadores de talla en nuestro país, como son José María Rey Benayas (20012004), Jordi Cortina (2004-2008) y Luis Navarro (2008-2012). Estos han sabido guiar con buen tino la dirección de la revista para el cumplimiento de sus objetivos y la han consolidado como un medio de comunicación en el campo de la ecología y el medio ambiente a nivel nacional e internacional, especialmente en Latinoamérica. El nuevo comité editorial, constituido por los autores de esta nota, tiene una visión continuista con respecto a lo que se ha venido haciendo hasta la fecha, pero con la firme intención de seguir mejorando la calidad de la revista. Para ello, hemos realizado una serie de cambios, tanto de contenidos como de forma, que esperamos sean del agrado de todos.

En lo referente a contenidos, hemos creído necesario hacer llegar el mensaje de que la revista no era, como a veces parecía desde fuera, una revista divulgativa científico-técnica, sino que con los años ya se había ido consolidando como una revista científica en el campo de la ecología y el medio ambiente. Y queremos que esta identidad quede muy clara entre los usuarios de la revista. El por qué de este cambio en el perfil de Ecosistemas está explicado con más detalle en otra nota editorial publicada en este mismo número (Cayuela et al. 2012). Además, hemos redefinido los tipos de contribuciones. Cada número se vertebrará en el contexto de un monográfico sobre una temática determinada. Los trabajos publicados dentro del monográfico serán propuestos por un/a Editor/a Invitado/a. Los autores que quieran contribuir con trabajos de temática diferente a la del monográfico, lo harán mediante envío directo para su revisión a la editorial bajo la forma de artículos de investigación, artículos de revisión o comunicaciones breves. También se siguen aceptando, como era tradición en la revista, resúmenes de tesis doctorales y, al igual que se hacía anteriormente, seguiremos otorgando un premio a la mejor tesis del año publicada en la revista. Las normas a los autores están ahora definidas con mayor precisión y exhaustividad, con objeto de facilitar el proceso de elaboración de los manuscritos por parte de los autores. Finalmente, se ha elaborado una política editorial en forma de criterios y preguntas, que será la base sobre la cual el comité editorial decidirá qué contribuciones serán publicadas en Ecosistemas (Cayuela et al. 2012). Esperamos que éstas hagan el proceso de edición más claro y transparente, y ayuden a los autores a decidir si su trabajo es o no idóneo para la revista.

En esta misma línea, hay un cambio notable en lo que respecta a la forma. Cualquiera se dará cuenta de que la revista tiene ahora una nueva apariencia. Ello es consecuencia de otro de los grandes cambios que presentamos: Ecosistemas se ha integrado dentro de la plataforma Open Journal System (OJS), que permite la gestión electrónica de manuscritos. A partir de ahora los autores que envíen sus trabajos a la revista lo harán por vía telemática (http://www.revistaecosistemas.net/index.php/ecosistemas/about/submissions\#o nlineSubmissions). Esto permitirá a editores, autores y revisores un ahorro de tiempo considerable, una gestión más eficiente en el proceso de publicación, así como una mayor trazabilidad de todos los pasos editoriales. Los artículos publicados en la revista ya no serán accesibles en formato $h t m l$, solo en formato $p d f$, si bien el título, autoría y resumen seguirán disponibles en $h t m l$, al modo convencional de la mayoría de las revistas de investigación. Como novedad importante los artículos tendrán a partir de este momento un 'DOI' (Digital Object Identifier), según el estándar de identificación de obras publicadas, ya común entre las revistas científicas internacionales. Este sistema ofrece una infraestructura técnica para el registro y uso de identificadores para su uso final en redes digitales, lo que hará más accesible y visible los artículos publicados en la revista.

Mantenemos obviamente nuestra política de acceso abierto, por creer firmemente que ésta contribuye a la difusión (aunque no necesariamente a la divulgación) de la ciencia (Navarro 2009). Además creemos que es necesario hacer permear la información generada a otros estratos de la sociedad, y la divulgación, ahora sí, se torna imprescindible en esta labor. Con este fin, hemos creado un blog asociado a la revista (http://revistaecosistemasblog.net), donde se divulgarán los contenidos de algunos de los trabajos más relevantes publicados en Ecosistemas. Para realizar esta labor hemos incorporado a nuestro equipo editorial a una Editora de Comunicación, Nieves Martín, especializada en labores divulgativas. 
Aparte de estos avances, nos hemos marcado en esta nueva etapa una serie de objetivos entre los que destacamos: (1) Obtener la indexación de la revista en Science Citation Index (SCI). Dados los contenidos que se publican en la revista creemos que este objetivo se podría conseguir a medio plazo, pero seguiremos requiriendo de la buena voluntad de los autores para que sigan enviando sus contribuciones a Ecosistemas. La indexación en SCI no es un fin en sí mismo, pero creemos que supondrá un mayor atractivo para los autores a la hora de enviar sus contribuciones a la revista, aumentando consiguientemente el nivel de calidad de los trabajos publicados. (2) Garantizar los contenidos de la revista a medio y largo plazo por medio de una cuidada planificación del trabajo. Queremos que esta premisa sea el punto de partida para organizar los contenidos de los próximos números y así, podemos anunciar ya el título de los dos primeros monográficos que publicaremos en 2013 bajo el nuevo formato de la revista. El primero, cuya Editora Invitada es Eva Hernández, del Instituto de Agricultura Sostenible (CSIC) de Córdoba, tratará sobre conservación de la biodiversidad en agrosistemas. El segundo, cuyos Editores Invitados son Jorge Cassinello y Lucía Gálvez, del Instituto de Recursos Cinegéticos (UCLM-CSIC) de Ciudad Real, abordará diversos temas relacionados con la gestión y conservación de recursos cinegéticos. Estos monográficos no solo están ya en marcha, sino que bastante avanzados, y esperamos que sean de interés para los lectores de Ecosistemas. (3) Agilizar el proceso de revisión. Pretendemos que el tiempo medio desde que un autor envía su trabajo a la revista hasta que recibe una respuesta, sea de 45 días. Creemos que el uso de la aplicación OJS nos ayudará en este sentido, aunque de nuevo necesitaremos la colaboración de toda la comunidad que rodea a Ecosistemas, ya que continuar con la buena voluntad y la excelente labor de los revisores sigue siendo el punto clave para conseguir esa mayor agilidad en el proceso.

Esperamos que todos estos cambios repercutan en un formato más atractivo para los lectores, pero también en un medio de comunicación más eficiente entre los autores y los editores, resultando finalmente en una revista de calidad en todos los sentidos.

\section{Vulnerabilidad de las especies al cambio climático}

Rodeado de todas estas novedades presentamos en este número un monográfico sobre la vulnerabilidad de las especies al cambio climático. Este monográfico contiene 12 artículos que analizan, desde distintas perspectivas metodológicas y enfoques, el efecto del cambio climático sobre diversas especies. La mayoría de los trabajos presentados se centran en la vulnerabilidad de la flora, fundamentalmente especies forestales, y se desarrollan mayoritariamente en la Península Ibérica. Todo ello confiere unas características únicas a este compendio de trabajos que esperamos sean del interés de los lectores.

El artículo de Felicísimo et al. (2012) presenta los resultados de un proyecto que desarrollaron los autores por encargo del Ministerio de Medio Ambiente y Medio Rural y Marino de España para evaluar la vulnerabilidad de 75 especies forestales y 145 de flora amenazada al cambio climático, utilizando para ello modelos de distribución potencial basados en el nicho climático actual y su proyección a escenarios futuros. En una línea temática afín, Arribas et al. (2012) presentan un trabajo de revisión en donde se discuten (con ejemplos) algunos conceptos fundamentales en este campo, como los factores que determinan la vulnerabilidad de una especie frente al cambio climático y su vinculación con propuestas de estrategias de gestión concretas.

Los trabajos de Linares et al. (2012), Martínez-Vilalta et al. (2012), Camarero et al. (2012), Sánchez-Salguero et al. (2012) y Candel Pérez et al. (2012) tienen un nexo común: todos ellos investigan el efecto directo e indirecto de la sequía sobre las poblaciones de distintas especies forestales en un contexto de cambio climático. El trabajo de Linares et al. (2012) estudia el efecto del cambio climático y el manejo del hábitat sobre el patrón de crecimiento y el actual declive observado en los cedrales del Atlas (bos- ques de Cedrus atlantica) en el norte de Marruecos. En el estudio de Martínez-Vilalta et al. (2012) se analizan diversas cuestiones relacionadas con las características ecofisiológicas que explican la vulnerabilidad del pino albar (Pinus sylvestris) a la sequía y los factores ambientales que determinan sus patrones de crecimiento y la variabilidad espacial en las tasas demográficas de la especie (crecimiento, mortalidad, reclutamiento) a distintas escalas. Camarero et al. (2012), por su parte, utilizan datos retrospectivos de crecimiento secundario, obtenidos mediante dendrocronología, y variables funcionales potencialmente indicadoras de cambios de vigor (frondosidad, producción de albura, cambios en las concentraciones de carbohidratos no estructurales en acículas y albura del tronco) para evaluar la respuesta de tres especies de árboles (Abies alba, Pinus sylvestris y Pinus halepensis) a la sequía extrema del año 2012. En esta misma línea, el trabajo de SánchezSalguero et al. (2012) analiza el impacto de diversas sequías recientes $(1994,1995,1999$ y 2005) sobre el crecimiento radial y el vigor de dos especies de pino (Pinus sylvestris, Pinus nigra) en zonas semiáridas del sudeste de la Península Ibérica (Sierra de los Filabres), cerca de su límite meridional de distribución. El estudio de Candel-Pérez et al. (2012) investiga los posibles efectos del cambio climático, caracterizado en la Península Ibérica por una disminución de las precipitaciones y el incremento de las temperaturas, sobre el crecimiento del pino laricio (Pinus nigra ssp. salzmannii) en la Serranía de Cuenca. Para complementar estos trabajos, se presenta una excelente revisión sobre la vulnerabilidad y la resiliencia de los ecosistemas forestales a episodios extremos de sequía (Lloret 2012).

En otra línea metodológica, Fernández Cancio et al. (2012) presentan una interesante contribución a la evaluación de los posibles efectos futuros del cambio climático en la distribución de los alcornocales (Quercus suber L.) en España, y sugiere algunas medidas alternativas de gestión. Para ello utilizaron una aproximación fitoclimática en función de 39 variables (periodo 1940-2005) para definir la idoneidad actual de la especie y aplicarlo en una proyección a 2050 según cuatro escenarios de cambio climático, evaluando la futura idoneidad del territorio. Los resultados de este estudio permiten definir zonas en donde en el futuro se reducirá el área de distribución actual del alcornoque (p.ej. Andalucía, Cataluña y Levante) y zonas que, por sus nuevas condiciones climáticas, serán aptas para el alcornoque y hacia donde se podrá expandir (p.ej. Galicia y Cornisa Cantábrica) siempre que se den las condiciones adecuadas para que se produzca su migración (existencia de corredores con una edafología adecuada).

El trabajo de Escudero et al. (2012) resume los resultados de varios estudios realizados por los autores en los pastos psicroxerófilos de la Sierra de Guadarrama para caracterizar sus respuestas al cambio global y evaluar su grado de vulnerabilidad. Sus resultados revelan un efecto del cambio climático sobre las especies de la alta montaña mediterránea, si bien las respuestas se manifiestan de manera muy diversa en distintas especies y a distintas escalas (nivel poblacional, nivel de comunidad). En la misma línea temática, Matías (2012) presenta una revisión de los últimos avances en el estudio de la dinámica poblacional de especies arbóreas en sus límites altitudinales y latitudinales y cómo su distribución se ve afectada por los cambios en el patrón climático. Además, analiza cómo los efectos de las variaciones en el clima pueden actuar conjuntamente con otros efectos indirectos, como es el caso de las alteraciones en algunas interacciones bióticas, entre las que destacan los cambios de distribución y presión de plagas, patógenos o herbívoros

Para finalizar este monográfico, Hódar et al. (2012), usando como hilo conductor a la procesionaria del pino (Thaumetopoea pityocampa), señala la necesidad de un abordaje más integral para la futura gestión de las plagas forestales. Mientras que el efecto del clima es reconocido (la procesionaria, por ejemplo, se ve afectada de forma positiva por los incrementos de temperatura durante el invierno), se muestran las posibilidades de una estrategia de prevención basada en el manejo del hábitat, que consiga bosques más 
diversos en estructura y composición específica, más resistentes y resilientes no sólo frente a las plagas, sino a los diversos tipos de perturbación que se adivinan para los años venideros.

Estamos seguros de que los lectores disfrutarán de este excelente compendio de artículos que tratan sobre un tema tan actual como controvertido, pero que los autores de estos trabajos han sabido abordar de una forma contundente y objetiva.

\section{El Comité Editorial de Ecosistemas}

Diciembre de 2012

\section{Referencias}

Arribas, P., Abellán, P., Velasco, J., Bilton, D.T., Lobo, J.M., Millán, A., Sánchez-Fernández, D. 2012. La vulnerabilidad de las especies frente al cambio climático, un reto urgente para la conservación de la biodiversidad. Ecosistemas 21(3):79-84.

Camarero, J.J., Sangüesa Barreda, G., Alla, A.Q,. González de Andrés, E., Maestro Martínez, M.,Vicente-Serrano, S.V. (2012). Los precedentes y las respuestas de los árboles a sequías extremas revelan los procesos involucrados en el decaimiento de bosques mediterráneos de coníferas. Ecosistemas 21(3):22-30.

Candel-Pérez, D., Lucas-Borja, M.E., Linares, J.C. 2012. Predicciones del crecimiento en poblaciones de pino laricio (Pinus nigra Arn. ssp. salzmanii) bajo diferentes escenarios futuros de cambio climático. Ecosistemas 21(3):41-49.

Cayuela, L., Alonso, A., Alonso, Blanco, J.A., Puerta-Piñero, C., Rodríguez Echeverría, S., Martín, N., Jiménez-Eguizabal, L. 2012. La política editorial de Ecosistemas. Ecosistemas 21(3):100-102.

Escudero, A., García-Camacho, R., García-Fernández, A., Gavilán, R.G., Giménez-Benavides, L., Iriondo, J.M., Lara-Romero, C., Morente, J., Pes- cador, D.S. (2012). Vulnerabilidad al cambio global en la alta montaña mediterránea. Ecosistemas 21(3):63-72.

Felicísimo, A.M., Muñoz, J., Mateo, R.G., Villalba, C.J. 2012. Vulnerabilidad de la flora y vegetación españolas ante el cambio climático. Ecosistemas 21(3):1-6.

Fernández Cancio, A., Sánchez-Salguero, R., Gil, P.M., Manrique Menéndez, E., Fernández Fernández, R., Navarro Cerrillo, R.M. 2012. Efectos del cambio climático sobre la distribución de los alcornocales españoles. Una aproximación fitoclimática para la futura gestión. Ecosistemas 21(3):50-62.

Hódar, J.A., Zamora, R., Cayuela, L. 2012. Cambio climático y plagas: algo más que el clima. Ecosistemas 21(3):73-78.

Linares, J.C., Pazo Sarria, R., Taïqui, L., Camarero, J.J., Ochoa, V., Lechuga, V., Seco, J.I., Viñegla, B., Sangüesa-Barreda, G., Gilarte, P., Merino, J., Carreira, J.A. 2012. Efectos de las tendencias climáticas y la degradación del hábitat sobre el decaimiento de los cedrales (Cedrus atlantica) del norte de Marruecos. Ecosistemas 21(3):7-14.

Lloret, F. 2012. Vulnerabilidad y resiliencia de los ecosistemas terrestres frente a episodios extremos de sequía. Ecosistemas 21(3):85-90.

Martínez-Vilalta, J., Aguadé, D., Banqué, M., Barba, J., Curiel Yuste, J., Galiano, D., Garcia, N., Gómez, M., Hereş, A.M., López, B.C., Lloret, F., Poyatos, R., Retana, J., Sus, O., Vayreda, J., Vilà-Cabrera, A. 2012. Las poblaciones ibéricas de pino albar ante el cambio climático: con la muerte en los talones. Ecosistemas 21(3):15-21.

Matías, L. 2012. Cambios en los límites de distribución de especies arbóreas como consecuencia de las variaciones climáticas. Ecosistemas. 21(3):91-96.

Navarro, L. 2009. La calidad de la información en las revistas de acceso abierto. Ecosistemas 18(2):1-2.

Sánchez-Salguero, R., Navarro-Cerrillo, R.M., Camarero, J.J., FernándezCancio, A., Swetnam, T.W., Zavala, M.A. 2012. Vulnerabilidad frente a la sequía de repoblaciones de dos especies de pinos en su límite meridional en Europa. Ecosistemas 21(3):31-40. 